\title{
Facial Recognition based on Histogram Matching with Adaptive Threshold
}

\author{
Luong Anh Tuan Nguyen \\ Ho Chi Minh City University of Transport \\ No.2, D3 Street, Ward 25, Binh Thanh District \\ Ho Chi Minh City, Vietnam
}

\begin{abstract}
Facial recognition was a field that was extensively studied in the past years but it is still an active area of research. This paper proposes a new method by matching histogram with adaptive threshold. The proposed method is simple but effective and it can be use for real-time system. Publicly available AT\&T database is used for the evaluation of the proposed method, which is consisted of 40 subjects with 10 images per subject containing variations in lighting, posing, and expressions. The proposed method provides a recognition rate higher than $99 \%$ and a verification error lower than $1 \%$.
\end{abstract}

\section{General Terms}

Facial recognition, Histogram

\section{Keywords}

Facial Recognition, Histogram, Histogram Matching, CrossCorrelation Coefficient, Adaptive Threshold

\section{INTRODUCTION}

Facial recognition is one of the central problems in computer vision. Many solutions have been proposed for solving facial recognition problem [1, 2, 3] due to its potential applications in many fields such as law enforcement applications, security applications and video indexing, etc. Several facial recognition systems have already been deployed at ports of entry at international airports in Australia and Portugal [4], most of them provides fairly good recognition rates although presents several limitations due to the illumination conditions.

Facial recognition solutions can be divided to two categories, such as, statistics-based and structure-based methods. For statistics-based method [1, 3, 5, 6], the face is treated as a two dimensional pattern of intensity variation. Under this method, the face is matched through finding its underlying statistical regularities. This method give the good facial recognition result. However, it is highly complicated for computation, making it difficult to implement them into real-time facial recognition applications. Structure-based methods [7, 8] uses the relationship between facial features, such as the locations of eye, mouth and nose. It can implement very fast, but recognition rate usually depends on the location accuracy of facial features, so it cannot give a satisfied recognition result. In this paper, a new method is proposed for facial recognition by matching histogram with adaptive threshold aims to overcome the disadvantages of statistics-based method. This paper is organized as follows: Section 2 presents in term of the theoretical background, and Section 3 shows the proposed method. Experimental results are presented in Section 4 and the conclusion is given in the last section.

\section{THE THEORETICAL BACKGROUND}

\subsection{Color Image}

Color image is presented by a triple RGB (Red, Green, Blue). The value of color channels ranges from 0 to 255 . Set of 3 color channels will generate $2^{24}$ colors $(256 * 256 * 256$ colors).

\subsection{Grayscale Image}

Grayscale images [9 10] are the color images using RGB color system in which the Red, Green, Blue have the same light intensity. So, the grayscale image just need to use one light intensity to show each pixel. The gray level of grayscale image ranges from 0 to 255 .

\subsection{Convert Color Image into Grayscale image}

There are some techniques to convert color image into grayscale image such as lightness with the equation $(\max (R, G, B)+$ $\min (\mathrm{R}, \mathrm{G}, \mathrm{B})) / 2$, luminosity with the equation $0.21 \mathrm{R}+0.72 \mathrm{G}$ $+0.07 \mathrm{~B}$. In this paper, the average method is selected, so the value of pixel in grayscale image is defined as (1).

$$
V=(R+G+B) / 3
$$

\subsection{Histogram}

Histogram [9, 11, 12] is the chart that shows the frequency of occurrence of each gray level in an image. Calculating histogram of image is performed as follows:

(1) Building the pixel matrix of the grayscale image.

(2) From the pixel matrix of the grayscale image, building the frequency of pixel.

Figure 1 presents the procedure of calculating the histogram of the grayscale image.

\subsection{Histogram of facial image}

Observing Figure 2, 3 and 4 it could found that the histograms of facial images of the same person are similarity. So, histogrambased facial recognition will be performed by matching between the histogram of testing facial image and the histogram of stored facial image.

\subsection{Histogram Matching}

In order to match histogram, cross correlation coefficient was used to calculate the similarity of histograms. The cross correlation coefficient [13, 14] is defined as [2]

$$
R_{x y}=\frac{\sum_{i=1}^{N}\left(X_{i}-\bar{X}\right)\left(Y_{i}-\bar{Y}\right)}{\sqrt{\sum_{i=1}^{N}\left(X_{i}-\bar{X}\right)^{2} \sum_{i=1}^{N}\left(Y_{i}-\bar{Y}\right)^{2}}}
$$

Where, $X_{i}$ and $Y_{i}$ are the $i^{\text {th }}$ element of two histogram vectors; $\mathrm{N}$ is the size of histogram vector, $\mathrm{N}$ is also called the number of 

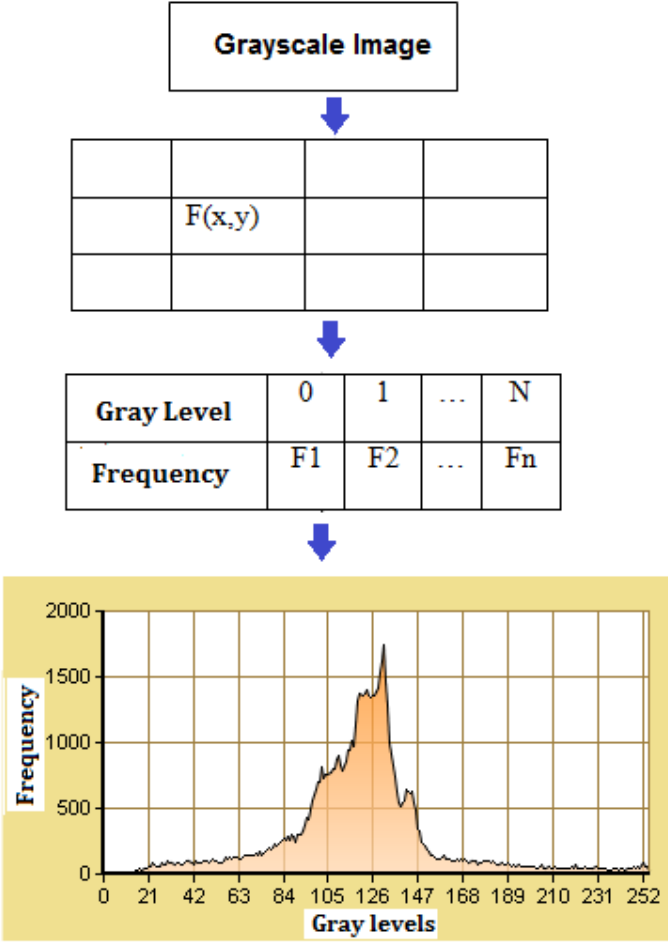

Fig. 1. The procedure of calculating the histogram of grayscale image.
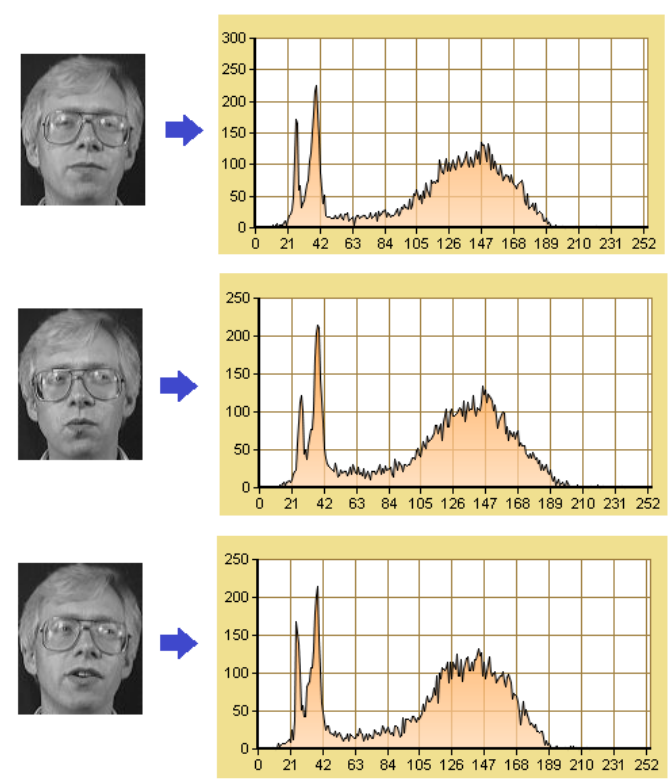

Fig. 2. Facial images with their corresponding gray level histograms.

bins (In this paper, $\mathrm{N}$ is 256). $\bar{X}$ and $\bar{Y}$ are the mean of the $\mathrm{X}$, $\mathrm{Y}$ vectors. $R_{x y}$ is cross correlation coefficient between the histogram of object $\mathrm{X}$ and the histogram of obect $\mathrm{X} . R_{x y}$ ranges from 0 to $1 . R_{x y}$ of 1 indicates maximum correlation and 0 indicating no correlation

\section{SYSTEM ARCHITECTURE}

System architecture consists of two phases: the training phase and the testing phase.
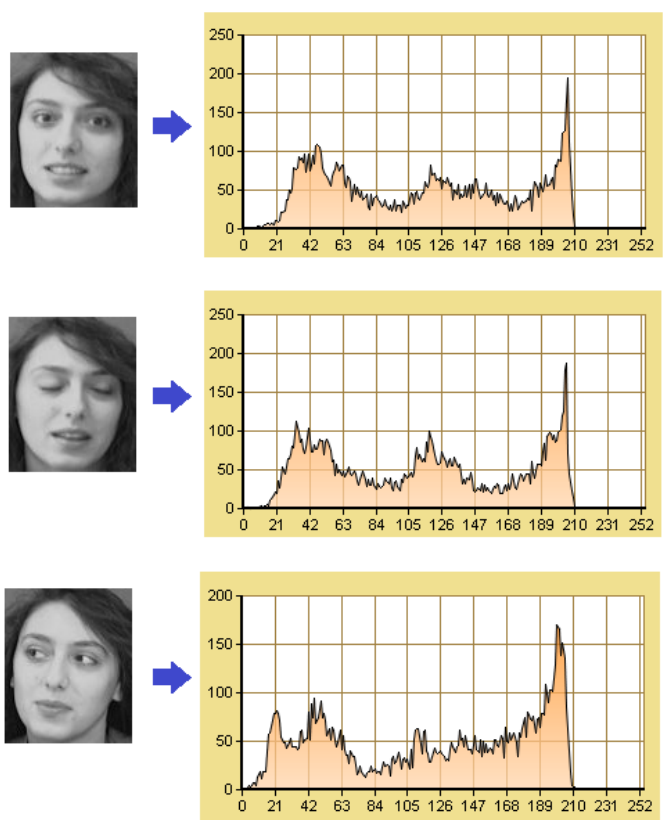

Fig. 3. Facial images with their corresponding gray level histograms.
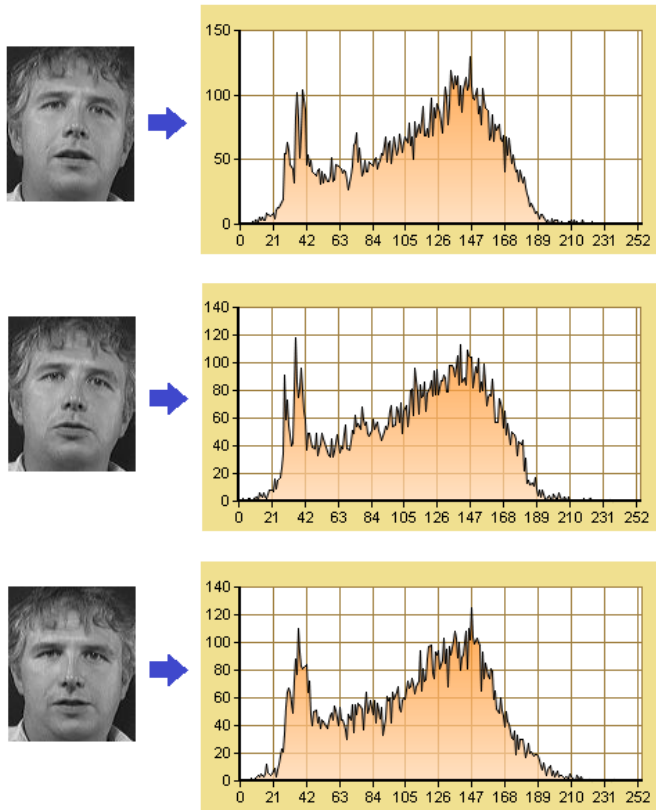

Fig. 4. Facial images with their corresponding gray level histograms

\subsection{The training phase}

The training phase is depicted in Figure 5 . The training phase is performed as follows:

(1) Get training facial image from disk or other resources.

(2) If training image is color image, it is converted into grayscale image.

(3) Get the histogram of grayscale image.

(4) Save the histogram of grayscale image into database.

\subsection{The testing phase}

The testing phase is depicted in Figure 6. The testing phase is performed as follows: 


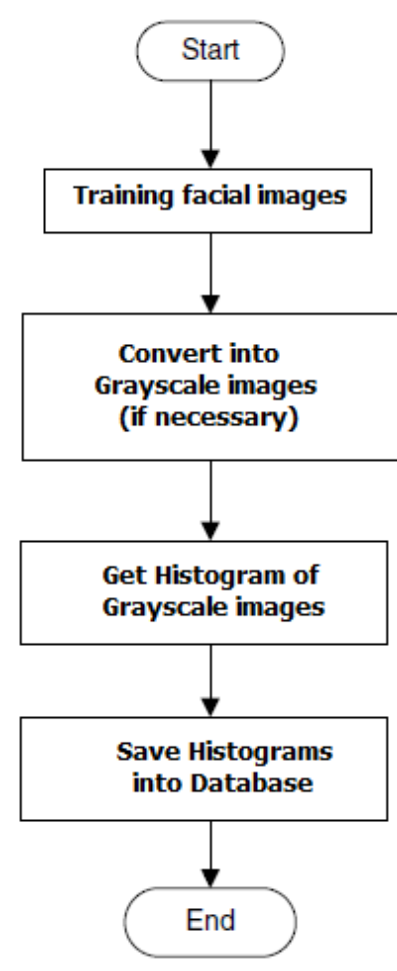

Fig. 5. The process of the training phase.

(1) Get testing facial image from disk or camera.

(2) If testing image is not facial image, the system is end. If testing image is facial image, the system goes on the next step.

(3) Extracting facial image from testing image.

(4) If extracted facial image is color image, it is converted into grayscale image.

(5) Get the histogram of grayscale image.

(6) Matching histogram aim to get the maximum cross correlation coefficient (Rmax).

(7) Comparing Rmax to the threshold to recognize face. The threshold is defined in section 3.3 .

\subsection{Adaptive Threshold}

Using the fixed threshold is simple, but the recognition result will be not effective. Using threshold adapting with dataset is complicated calculations but effectively.

In order to identify the threshold, the dataset consist of 10 facial images for each of $\mathrm{N}$ persons, 10 facial images were divided into 9 facial images for training and one facial image for testing.

The adaptive threshold is identified as follows:

(1) Get the histograms of 9 training facial images for each person.

(2) Get the histogram of testing facial image for each person.

(3) Calculating cross correlation coefficient between the histogram of testing facial image and the histograms of 9 training facial images for each person.

(4) Get minimum cross correlation coefficient from 9 cross correlation coefficients for each person $\left(\operatorname{Rmin}_{i}, \mathrm{i}=1 . . \mathrm{N}\right)$

(5) The threshold is minimum cross correlation coefficient for $\mathrm{N}$ person from $\mathrm{N}$ minimum cross correlation coefficients above. The threshold is defined as 3 :

$$
\text { Threshold }=\min _{1 \leq i \leq N}\left(\operatorname{Rmin}_{i}\right)
$$

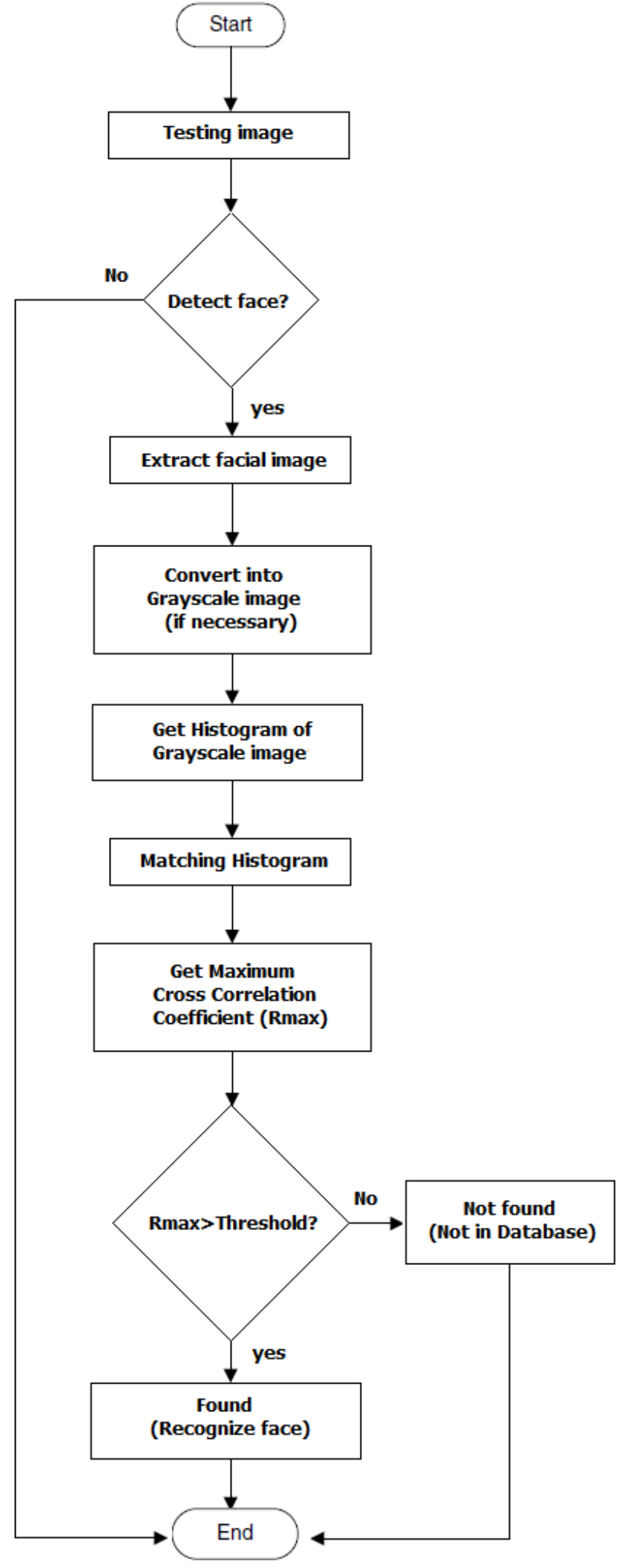

Fig. 6. The process of the testing phase. 


\section{EXPERIMENTAL RESULTS}

\subsection{Dataset}

Face database of AT\&T Laboratories Cambridge [15] is used for recognition experiments. In the database, 10 facial images for each of 40 persons (totally 400 images) with variations in face angles, face sizes, facial expressions, and lighting conditions are included. Each image has a resolution of $92 \times 112$. Face database of AT\&T Laboratories Cambridge is also called ORL database.

\subsection{Experiment 1}

In this experiment, the three subsets are organized as follows:

(1) In the first subset, 10 facial images for each person consist of 3 facial images for training and 7 facial images for testing.

(2) In the second subset, 10 facial images for each person consist of 5 facial images for training and 5 facial images for testing.

(3) In the third subset, 10 facial images for each person consist of 7 facial images for training and 3 facial images for testing.

The experimental process for training is performed as follows:

(1) Get the histogram of each training image and save it into the database.

(2) Determining the threshold for each subset and save it into the database.

The experimental process for testing is performed as follows:

(1) Get the histogram of each testing image.

(2) For each testing image,

(a) Calculating cross correlation coefficient between the histogram of the testing image and the histogram of each training image.

(b) Get maximum cross correlation coefficient for each person in database (Rmax 1, Rmax2,..,Rmax40)).

(c) Get maximum cross correlation coefficient for 40 person in database (Rmax) by (4):

$$
R \max =\max _{1 \leq i \leq 40}(R \max i)
$$

(d) Comparing Rmax to the threshold to give the result of recognition.

The proposed method uses root mean square error (RMSE) [16] to measure recognizing accuracy. The correct recognition rates in percent are included in Table 1 Each result is the average of 100 runs, where we have randomly shuffled the facial image in each run. Figure 7 shows the detail results of 100 runs.

\subsection{Experiment 2}

In this experiment, the three subsets are organized like the first experiment.

The experimental process for training is performed as follows:

(1) Get the histogram of each training image.

(2) Calculating the average value of histograms for each class (each person) and save it into database.

(3) Determining the threshold for each subset and save it into the database.

Table 1. The experimental results of the first experiment.

\begin{tabular}{|c|c|c|}
\hline Subset & Recognition rates & Time for recognizing a facial image (ms) \\
\hline $1^{\text {st }}$ & $94.76 \%$ & 534 \\
\hline $2^{\text {nd }}$ & $97.77 \%$ & 783 \\
\hline $3^{\text {rd }}$ & $99.48 \%$ & 1176 \\
\hline
\end{tabular}

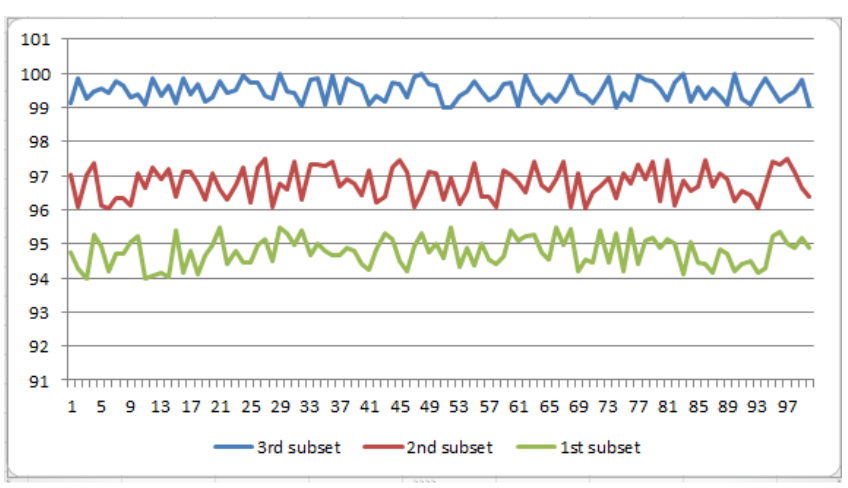

Fig. 7. The detail results of 100 runs in the first experiment.

Table 2. The experimental results of the second experiment.

\begin{tabular}{|c|c|c|}
\hline Subset & Recognition rates & Time for recognizing a facial image (ms) \\
\hline $1^{\text {st }}$ & $92.73 \%$ & 121 \\
\hline $2^{\text {nd }}$ & $94.70 \%$ & 217 \\
\hline $3^{\text {rd }}$ & $97.91 \%$ & 308 \\
\hline
\end{tabular}

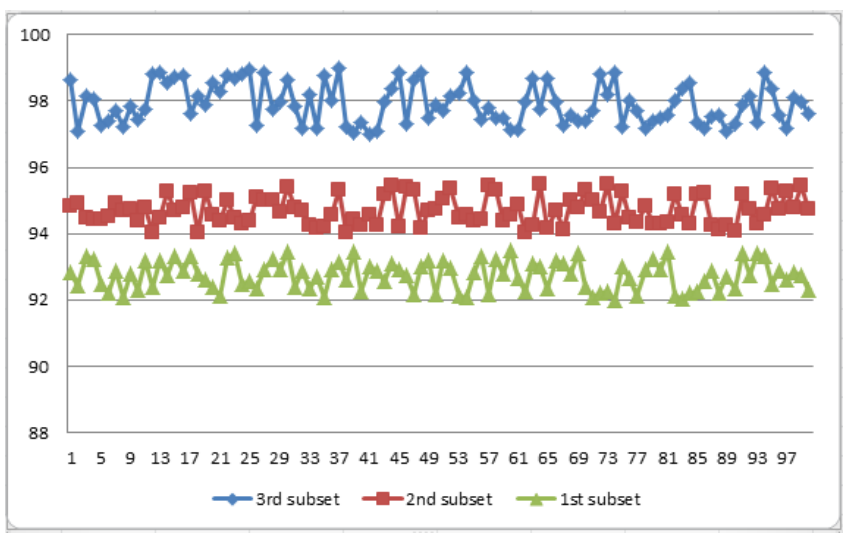

Fig. 8. The detail results of 100 runs in the second experiment.

The experimental process for testing is performed as follows:

(1) Get the histogram of each testing image.

(2) For each testing image,

(a) Calculating cross correlation coefficient between the histogram of the testing image and the average histogram of each person (R1, R2, .., R40).

(b) Get maximum cross correlation coefficient for 40 person in database ( $\operatorname{Rmax})$ by (5):

$$
R \max =\max _{1 \leq i \leq 40}(R i)
$$

(c) Comparing Rmax to the threshold to give the result of recognition.

The correct recognition rates in percent are included in Table 2 Each result is the average of 100 runs, where we have randomly shuffled the facial image in each run. Figure 8 shows the detail results of 100 runs. Comparing to the first experiment, the second experimental result decreased effective slightly, but the time of recognition decreased significantly.

\subsection{Comparing to other works}

The performance of PCA and LDA based facial recognition systems in the same setup [6] is lower than the proposed method. Table 3 shows the results of the comparison between the second 
Table 3. The results of the comparison to other works.

\begin{tabular}{|c|c|c|c|}
\hline Subset & PCA & LDA & The proposed method \\
\hline $1^{\text {st }}$ (3 training facial images) & $73 \%$ & $82 \%$ & $92.73 \%$ \\
\hline $2^{\text {nd }}$ (5 training facial images) & $78 \%$ & $87 \%$ & $94.70 \%$ \\
\hline $3^{\text {rd }}$ (7 training facial images) & $92 \%$ & $95 \%$ & $97.91 \%$ \\
\hline
\end{tabular}

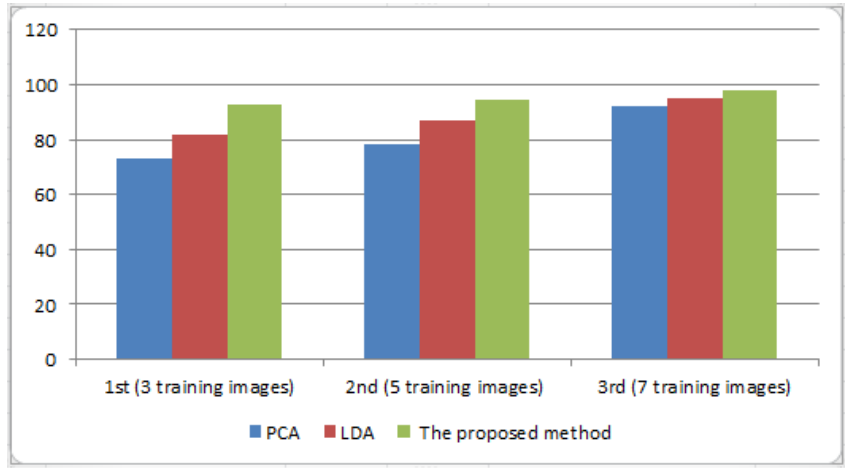

Fig. 9. The results of the comparison.

experiment and the work [6]. The results of the comparison are depicted in Figure 9

\section{ACKNOWLEDGMENTS}

The authors wish to thank the authors of ORL database and thank reviewers for their reading of our manuscript and their insightful comments and suggestions.

\section{CONCLUSIONS}

This paper proposed a new method using histogram matching with adaptive threshold for facial recognition. The proposed method was experimented with two other experiments by using publicly available ORL database. A very high recognition rate is achieved by using the proposed method. The best recognition rate reaches to $99.48 \%$. Furthermore, in the second experiment, the best recognition rate also reaches to $97.91 \%$ and the time of recognition decreased significantly. This is a good idea to build a real-time system for facial recognition. The results of the proposed method were also compared to other works aim to evaluate the effectiveness of the proposed method.

In the future, The proposed method will be improved in the manner of the second experiment. We will experiment with many other datasets aim to improve the calculation of the crosscorrelation coefficient, the calculation of the adaptive threshold and increasing the effectiveness of facial recognition further.

\section{REFERENCES}

[1] M.A. Turk, and A.P. Pentland, Face recognition using eigenfaces, in Proc. IEEE Computer Society Conf. on Computer Vision and Pattern Recognition, 1991, pp. 586591 .

[2] S. G. Karungaru, M. Fukumi, and N. Akamatsu, Face recognition in colour images using neural networks and genetic algorithms, Intl Journal of Computational Intelligence and Applications, vol. 5, no. 1, pp. 55-67, 2005.

[3] K. Kotani, Q. Chen, F. F. Lee, and T. Ohmi, Regiondivision VQ histogram method for human face recognition, Intelligent Automation and Soft Computing, vol. 12, no. 3, pp. 257-268, 2006.
[4] R. Chellapa, P. Sinha, P. J. Phillips, Face recognition by computers and humans, Computer Magazine, Vol. 43, pp. 46-55, Feb. 2010.

[5] Pierluigi Carcagni, Marco Del Coco, Marco Leo, Cosimo Distante, "Facial expression recognition and histograms of oriented gradients: a comprehensive survey" in Springer Open Journal, pp. 1-25, 2015

[6] Alaa Eleyan, Hasan Demirel, "PCA an LDA based face recognition using feedforward neural network classifier", Lecture Notes in Computer Science(MRSC 06), vol.4105, pp. 199 206, Jun. 2006.

[7] R. Brunelli and T. Poggio, Face recognition: features versus templates, IEEE Trans. Pattern Analysis and Machine Intelligence, vol. 15, no. 10, pp. 1042-1052, 1993.

[8] L. Wiskott, J. M. Fellous, N. Kruger, and C. Malsburg, "Face recognition by elastic bunch graph matching," IEEE Trans. Pattern Analysis and Machine Intelligence, vol. 19, no. 10, pp.775-780, 1997.

[9] Luong Anh Tuan Nguyen, Huu Khuong Nguyen. Traffic Density Identification Based On Histogram. Journal of Transportation Science and Technology, ISSN: 1859-4263, Vol 15-05/2015, pp 23-27.

[10] Xiangyun Ye, Mohamed Cheriet, Senior Member, Ching Y. Suen (2001), Stroke-Model-Based Character Extraction from Gray-Level Document Images, IEEE, 2001.

[11] C. C. Sun. S. J. Ruan, M. C. Shie, T. W. Pai, Dynamic Contrast Enhancement based on Histogram Specification, IEEE Transactions on Consumer Electronics, 51(4), pp.13001305, 2005.

[12] Luong Anh Tuan Nguyen, Thi-Ngoc-Thanh Nguyen. Traffic Image Classification using Horizontal Slice Algorithm. International Journal of Computer Applications (ISSN: 0975 8887), Volume 148 No.11, pp. 30-34, August 2016.

[13] Al Bovik, Handbook of Image and Video Processing, Academic Press, 2000.

[14] Gonzalez, R., C., and Woods, R., E., 2001, Digital Image Processing, Prentice Hall, NJ, 2001

[15] AT\&T Laboratories Cambridge. The database of faces. [Accessed September, 2017]. Available: http://www.cl.cam.ac.uk/research/dtg/attarchive/facedatabase.html

[16] C. Willmott, and K. Matsuura, Advantages of the Mean Absolute Error (MAE) over the Root Mean Square Error (RMSE) in assessing average model performance, Clim. Res., 30, 7982, 2005. 